\title{
O COTIDIANO DA VIDA DE HIPERTENSOS: MUDANÇAS, RESTRIÇÕES E REAÇÕES.
}

\section{HYPERTENSION'S DAILY LIVING: CHANGES, RESTRICTIONS AND REACTIONS.}

Vanda Dias de Castro* Marcia Regina Car**

CASTRO, V. D; CAR, M. R. O Cotidiano da vida de hipertensos: mudanças, restrições e reações. Rev.Esc.Enf.USP, v. 34, n. 2 , p. 145-53, jun. 2000.

\section{RESUMO}

Objetivou-se descrever o cotidiano da vida de hipertensos após o conhecimento do diagnóstico da hipertensão arterial (HA); e, identificar possiveis mudanças na dinâmica da vida de hipertensos após o inicio do tratamento da HA. Utilizouse o método descritivo com abordagem qualitativa e a análise de discurso dos depoimentos de seis hipertensos. Evidenciaram-se diferentes comportamentos/reações dos doentes em relação aos seus cotidianos após a descoberta da HA, nem sempre coincidentes no tempo, com o início do tratamento anti-hipertensivo. Essas diferenças parecem estar associadas à compreensão dos doentes sobre o significado da HA e ao impacto desta nas suas vidas. Em sintese, conviver com a cronicidade da HA significa, ao longo do tempo, fazer/sofrer mudanças no cotidiano da vida.

PALAVRAS-CHAVE:Doença crônica.Hipertensão.

\begin{abstract}
Aiming to describe daily living of hypertensive people after Arterial Hypertension (AH) diagnoses and to identify changes in their lives. The method of inquiry used was descritive and qualitative using discursive method to figure out theme factors. The results showed different behaviors/reactions of being sick according to their daily living after discovering AH, that not always happened at the same time that the antihypertensive treatment began. Those differences seems to be associated with individual understanding of sickness itself and of impact in their lives. So, getting along with hypertension is to learn about the sickness and symptoms and also make the necessaries changes.
\end{abstract}

KEYWORDS: Cronic disease. Hypertension.

\section{INTRODUÇÃO E OBJETIVOS}

A necessidade de tratamento, a longo prazo, da hipertensão arterial (HA) para a prevenção das complicações cérebro-cardiovasculares e renais, vem sendo estudada principalmente devido à complexidade que a envolve. Desde a organização e os recursos, humanos e materiais, dos serviços de saúde até a adesão dos hipertensos ao seu tratamento, têm sido investigadas, em geral, com abordagens quantitativas.

A doença crônica é um problema de saúde a longo prazo e quanto maior o tempo de exposição à doença, maior o comprometimento dos sistemas orgânicos (BLACK; MATASSARIN-JACOBS, 1996). Com isso, ela pode trazer complicações sérias para o indivíduo que, comprometem seu estado físico, causam incapacidades e exigem mudanças em seu estilo de vida. Seu controle adequado promove a redução de complicações e melhora na expectativa de vida (MENDES; TREVIZAN, 1981).

O tratamento de uma doença crônica é muito mais que um método para controlar sintomas, lidar com incapacidades ou adaptações para as mudanças

\footnotetext{
* Aluna do $8^{\circ}$ semestre do Curso de Graduação em Enfermagem da Escola de Enfermagem da Universidade de São Paulo.

** Professora Doutora do Departamento de Enfermagem Médico-Cirúrgica da Escola de Enfermagem da Universidade de São Paulo. E-mail marcrcar@usp.br
} 
psicológicas e sociais que uma doença incurável a longo prazo traz para a vida das pessoas atingidas e seus familiares, sendo um processo de mudança muito complicado (WOOG, 1992).

Principalmente no caso da hipertensão arterial, para que seu tratamento atinja a eficácia desejada que é a diminuição da morbidade e da mortalidade cardiovascular, se faz necessária a adoção de medidas que interferem no estilo de vida dos hipertensos e que, comprovadamente, favorecem a redução da pressão arterial. Tais medidas são: redução do peso corporal, da ingestão do sal e do consumo de bebidas alcóolicas; prática de exercícios físicos com regularidade; e a não utilização de drogas que elevam a pressão arterial. Há outras razões que tornam essas modificações no estilo de vida úteis, além da redução 'da pressão arterial que favorece o controle de outros fatores de risco: baixo custo e risco mínimo; aumento da eficácia do tratamento medicamentoso e redução do risco cardiovascular (III CONSENSO, 1998).

É importante lembrar que o indivíduo portador de uma doença crônica atravessa períodos de irritabilidade e autodepreciação, onde o processo de adaptação é lento e longo, e depende do tipo de doença, do grau de incapacidade e personalidade do indivíduo. Muitas vezes apresentam sentimentos de desamparo, desesperança, raiva, depressão, tristeza, solidão, que geram angústia e pesar (SILVA; COSTA; RIZZO, 1980).

Psicologicamente, o indivíduo trava uma luta consigo mesmo onde aceitar ou negar o diagnóstico implicará em enfrentamento ou fuga da morte. Cada indivíduo utiliza um determinado tipo de mecanismo que auxilia na adaptação à doença. Há três fases que caracterizam bem isso: a $1^{\text {a }}$ fase - de resistência, onde ocorre a negação da doença; a 2a fase - de conscientização, onde há a consciência e lamentação da doença através de depressão, choro, raiva dos demais, etc; e a $3^{\text {a }}$ fase - de aceitação, com a adaptação ao novo e equilíbrio das emoções. Socialmente, a doença crônica afeta a função ou o papel que o individuo desempenha perante os demais, de acordo com os valores, crenças e a cultura de cada um, e, conseqüentemente, afeta os demais setores sociais como economia, educação, trabalho e lazer (BLACK; MATASSARIN-JACOBS, 1996).

O processo de adaptação às modificações no estilo de vida, em se tratando de hipertensos, é bastante complexo e relaciona-se diretamente ao seguimento do tratamento para o controle da hipertensão arterial. Fatores como "desconhecimento individual do diagnóstico, a disponibilidade quantitativa e qualitativa da assistência médica e o acesso aos serviços de saúde", interferem na eficácia e eficiência do tratamento da hipertensão arterial. A adesão ao tratamento implica em dificuldades como ingestão de dieta hipossódica, dinheiro para a compra de medicamentos e para o transporte até o serviço de saúde, além da distância para chegar até o mesmo. Quanto às facilidades que contribuiriam para a adesão ao tratamento encontram-se o recebimento gratuito de medicamentos da instituição, a facilidade de marcar consultas, a proximidade do serviço de saúde, o fornecimento de vale transporte e o apoio familiar. O abandono do tratamento é uma das grandes dificuldades para o seguimento do tratamento da hipertensão artrial e está relacionado a uma significante insatisfação com as modificações do estilo de vida dos hipertensos e/ou recursos financeiros, em sua maioria (CASTRO; CAR, 1997).

Entendendo-se a cronicidade da hipertensão arterial e de seu tratamento como fatores intervenientes no processo de vida dos hipertensos, este estudo tem por objetivos:

- Descrever o cotidiano da vida de hipertensos após o conhecimento do diagnóstico da hipertensão arterial;

- Identificar possiveis mudanças na dinâmica da vida de hipertensos após o início do tratamento da hipertensão arterial.

\section{PERCURSO METODOLÓGICO}

\section{Método}

Este é um estudo descritivo exploratório, com uma abordagem qualitativa.

\section{Sujeitos da Investigação}

Fizeram parte do estudo seis hipertensos em tratamento da HA, há pelo menos seis meses, e, que no momento, estavam hospitalizados em uma instituição pública universitária de grande porte, na cidade de São Paulo, para esclarecimento do diagnóstico ou por complicações decorrentes da hipertensão arterial.

\section{Coleta de Dados}

No primeiro momento realizou-se a seleção dos doentes, internados no mês de outubro de 1998, compativeis com os critérios de inclusão no estudo, em seguida foi solicitado o consentimento informado, por escrito, do hipertenso para sua participação no estudo.

Após seu consentimento, cada hipertenso foi submetido a uma entrevista, gravada, com as seguintes questões norteadoras, nessa ordem (a segunda questão só foi feita após a resposta da primeira, para que não houvesse influência da segunda questão sobre a primeira): 
- Como vem sendo a sua vida desde que descobriu que precisava tratar a pressão alta?

- Houve mudanças na sua vida após o início do tratamento da pressão alta?

Em seguida foram obtidos os dados de identificação e caracterização social dos hipertensos (Anexo).

\section{Tratamento dos Dados}

As entrevistas foram transcritas na integra e submetidas à técnica de análise de discurso preconizada por FIORIN; SAVIOLI (1991) e adaptada por CAR (1993). As frases temáticas, depreendidas dos discursos dos hipertensos, foram categorizadas e analisadas em função da relação entre o desenvolvimento do cotidiano da vida dos hipertensos e a cronicidade da HA.
Desta forma, a partir da análise dos depoimentos foram identificados cinco temas centrais: a vida após a descoberta da HA; a descoberta do diagnóstico da $\mathrm{HA}$; a compreensão do significado da HA; as dificuldades no seguimento do tratamento da HA e mudanças na vida dos doentes após o início do tratamento da HA, apresentados na discussão a seguir.

\section{RESULTADOS E DISCUSSÃO}

\subsection{Identificação e Caracterização Social dos doentes}

A amostra constituída por 6 hipertensos hospitalizados, caracterizou-se conforme apresentase no quadro 1.

\section{Quadro 1- Dados de Identificação e Caracterização Social dos doentes.}

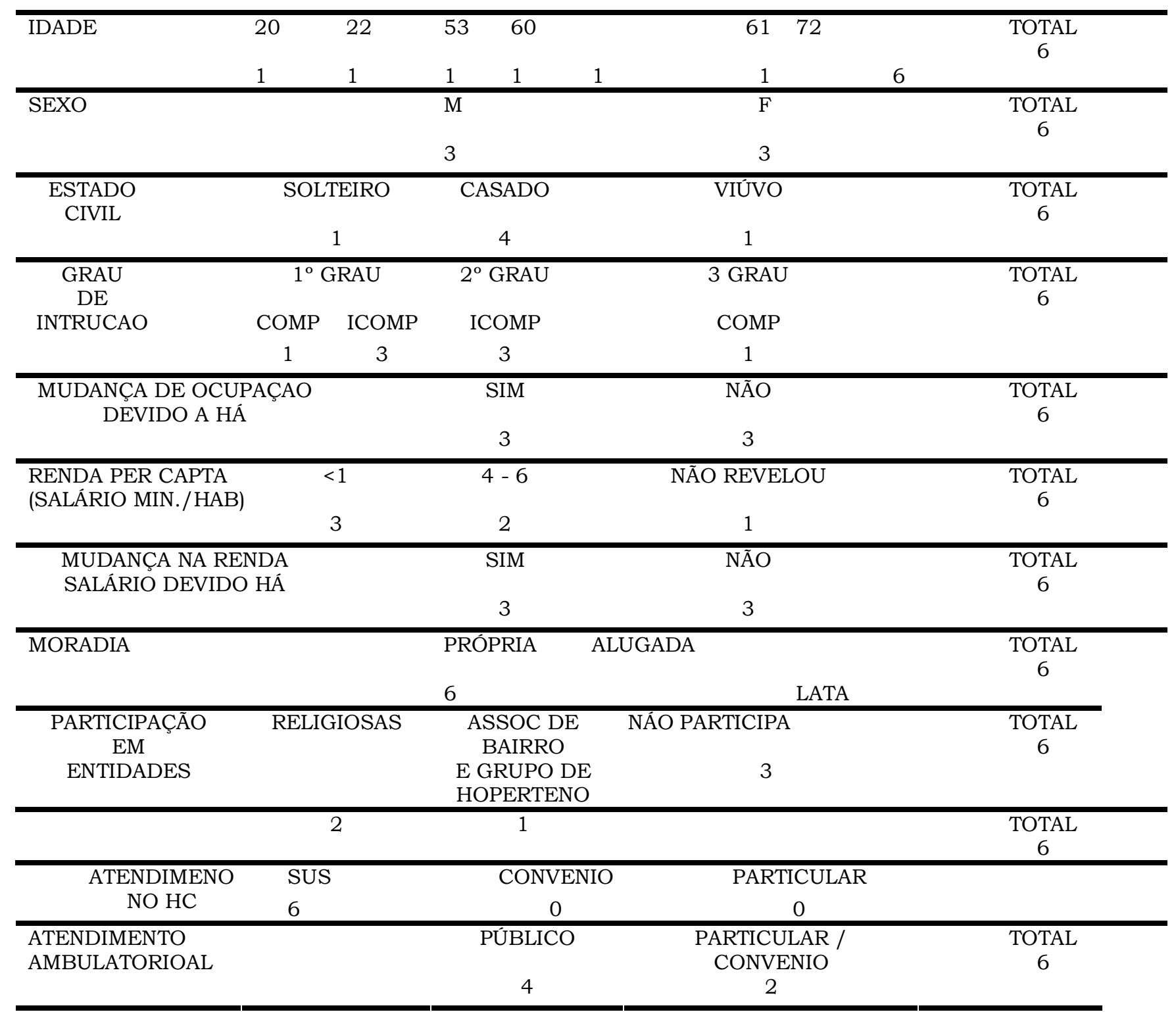


O grupo de hipertensos participantes deste estudo (6) caracterizou-se pela diversidade na idade e na escolaridade. Segmentou-se na metade em relação ao sexo, à renda per capta, à participação em entidades/grupos e às mudanças de ocupação e de renda devido à HA. Apesar destas características diversas houve homogeneidade no grupo quanto a dependência do serviço público de saúde, tanto para a hospitalização, quanto para o seguimento ambulatorial.

\subsection{0 cotidiano da vida dos hipertensos}

\subsubsection{A vida após a descoberta da hipertensão arterial}

Em relação ao cotidiano da vida, após a descoberta da hipertensão arterial, houve diferentes comportamentos/reações entre os doentes estudados: a manutenção da "normalidade" da vida, sem nenhuma modificação, mesmo quando os hábitos eram considerados "exageros"; o simples acréscimo da tomada de medicação diária até a manifestação de sentimentos de "revolta" e "raiva"; e, a preocupação por parte de familiares e amigos com o doente. As frases temáticas que expressam tais reações são:

A descoberta da hipertensão arterial não modificou o cotidiano da vida do doente ("não mudou nada... fazia os mesmos exageros que sempre fiz: sempre fumei, sempre bebi... nunca dei importância a comer sem sal");

Não houve modificações na vida do doente após a descoberta da hipertensão arterial ("perfeitamente normal... absolutamente normal... tomo o meu remedinho de pressão, isso pra mim não chega a ser nada de chocante assim...") exceto pela tomada de medicação;

A vida após a descoberta da hipertensão arterial se tornou "uma tragédia... muito dificil... muito sofrimento, muita atribulação, revoltada, eu não conseguia fazer tudo o que eu costumava fazer";

A preocupação com a doente por parte da mãe, dos médicos e dos amigos é vista como "dó", o que provocava "raiva" na doente ("eu achava horrivel").

Uma doente justificou, através da fatalidade, a manutenção da "normalidade" de seu cotidiano da vida:

A descoberta da hipertensão arterial não provocou "medo" nem "revolta" por ter sido considerada um acontecimento da vida e atribuída à vontade de "Deus".

\subsubsection{A descoberta do diagnóstico da hipertensão arterial}

A descoberta do diagnóstico da hipertensão arterial está relacionada à presença de sintomas que levaram à procura de atendimento de saúde em local de trabalho, unidade básica de saúde e serviço de emergência. Apenas um doente descobriu ser hipertenso através de um "check up" de rotina. As frases temáticas a seguir, ilustram essa discussão:

A descoberta do diagnóstico da hipertensão arterial está relacionada à presença de sintomas (vômito, cefaléia, foto fobia e edema facial);

A medida da pressão arterial alterada foi realizada pela enfermeira (do trabalho) que encaminhou o doente para orientação médica;

O diagnóstico da hipertensão arterial foi feito, há 19 anos, em um atendimento de emergência devido ao "problema alcóolico" do doente;

A descoberta do diagnóstico da hipertensão arterial, pelo doente, se deu através da medida da pressão arterial em um "check up" de rotina;

A descoberta da hipertensão arterial, há 4 anos, está relacionada à presença de sintomas (cefaléia intensa), sendo que o diagnóstico foi feito pelo médico no local de trabalho;

A descoberta da hipertensão arterial, há 3 anos, se deu através do aparecimento de sintomas ("adormecia os braços, muita dor de cabeça, vista ruim").

Com relação à sintomatologia da HA, o estudo de PESSUTO (1994) revelou que, apesar da HA ser considerada em geral assintomática, uma pequena parcela de clientes referiu não apresentar sintomas. "A sintomatologia levantada mostrou que necessidades fisiológicas poderiam estar afetadas e pôde-se associá-las à falta de conhecimento em relação à patologia e ao próprio tratamento, que, caso este não seja seguido corretamente pode ser causador de alguns sintomas citados, indicando que complicações da HA podem estar se manifestando". Os sintomas mais mencionados foram: dor de cabeça, tontura, palpitação e dor na nuca.

O modelo de assistência à saúde dominante no nosso meio não vem privilegiando as ações de prevenção que promoveriam, entre outras, a detecção precoce das doenças. Assim, os hipertensos descobrem seu problema já numa fase onde complicações podem estar presentes, o que justificaria a sintomatologia referida. 
MACIEL (1997) refere que, em seu estudo, usuários verificavam a pressão arterial com maior freqüência no posto de saúde, seguido da farmácia; outros locais como hospitais, ambulatórios e residência apareceram e foram citados em menor freqüência. Tendo esta investigação sido realizada na cidade de Ribeirão Preto - SP, pode estar apontando uma realidade de organização e procura da unidade básica de saúde diferente daquela da cidade de São Paulo onde residem os doentes do presente estudo.

\subsubsection{A compreensão do significado da hipertensão arterial}

A importância dada ao seguimento do tratamento da hipertensão arterial parece estar diretamente relacionada à compreensão que os doentes têm sobre a doença. Influenciaram estas compreensões a presença da hipertensão arterial na família, o fato de ser jovem não ser associado à doença, a medida numérica da pressão arterial elevada ser uma abstração que só se concretiza quando do aparecimento de complicações. Estas questões podem ser evidenciadas pelas frases temáticas a seguir:

Existe conhecimento do diagnóstico de hipertensão arterial há 4 anos e o tratamento foi iniciado há 2 anos, porque "a família tudo tem isso, não liguei";

A compreensão do significado da hipertensão arterial foi após 4 anos do seu diagnóstico, quando o doente teve o $1^{\circ}$ IAM ("aí... é que eu vim saber o que era ser hipertenso, era colesterol alto, era vida sedentária, era tabagismo...");

Para o doente a hipertensão arterial é uma medida numérica detectada pelo médico ("...uma coisa grega, você é hipertenso! Ah, bonito... poderia ser uma coisa de comer, ou de beber...");

A descoberta da hipertensão arterial, há 3 anos, foi desconsiderada pela doente porque ela não dava importância "a esse negócio de doença" e por ser jovem e desconhecer outros da sua idade com o mesmo problema.

As concepções de saúde-doença identificadas nos discursos dos doentes são divergentes. Um deles compreende a relação das formas de trabalhar e viver com o desenvolvimento da doença; enquanto outro acredita que não ter dor significa ausência de doença; e, outros associam a um fator de risco (ingestão de sódio) o descontrole da pressão arterial:

A manutenção da pressão arterial elevada foi associada ao não seguimento da dieta hipossódica;
O aparecimento da hipertensão arterial é associado, pelo doente, às formas de viver $e$ de trabalhar em uma grande metrópole ("agitada") que provavelmente levam a uma pessoa ter tendência à pressão alta;

O descontrole da hipertensão arterial foi associado ao não seguimento de dieta hipossódica (em casa) levando o médico a manter a doente internada;

A ausência de dor significa não estar doente.

Evidenciou-se também, nos depoimentos de doentes, o desconhecimento das complicações decorrentes da hipertensão arterial e também do seu tratamento:

As complicações cardiovasculares (2 IAMs e crises de Angina) e renais não são associadas, pelo doente, ao diagnóstico de hipertensão arterial há 19 anos;

A orientação da psicóloga de que "diálise não tem cura e é para a vida toda" provocou mais revolta na doente;

A doente pede a Deus para não fazer mais diálise (..."Deus tivesse misericórdia de mim, que um dia eu ia sair daquela máquina...") atribuindo ao Divino o provável sucesso da cirurgia.

Assim, a compreensão do significado da HA parece estar associada tanto a valores e crenças dos doentes quanto ao nível de conhecimento.

TRENTINI; SILVA; LEIMANN (1990) indicaram que o conhecimento sobre a doença é mais uma tarefa das novas incumbências atribuídas aos doentes crônicos, inclusive os hipertensos. Neste estudo uma parte dos doentes procurou saber sobre a doença e a outra não buscou informações, justificando que apesar de ter vontade de se informar não o fazia por temer uma doença indesejada ou por não gostar de perguntar.

O estudo de PESSUTO (1994) evidenciou que $54,3 \%$ dos hipertensos não sabiam se a HA estava relacionada a outras doenças; $15,7 \%$ afirmaram não haver relação alguma entre a HA e outras doenças; e, outros $12,8 \%$ associaram a HA à doenças renais, cardiacas, entre outras. "Pelo fato de a maioria $(54,3 \%)$ não saber a origem de sua hipertensão, supõe-se que haja a necessidade de maiores esclarecimentos sobre a patologia, ou porque os clientes foram orientados mas não ficaram esclarecidos, devido à uma inadequação por parte de um dos lados, ou porque os mesmos não receberam as orientações necessárias sobre a doença". 


\subsubsection{Dificuldades no seguimento do tratamento da hipertensão arterial}

Foram identificadas dificuldades no seguimento do tratamento da hipertensão arterial relacionadas à medicação, à dieta e à acessibilidade ao serviço de saúde.

Com relação à medicação, as justificativas apontadas pelos doentes para a tomada irregular dos medicamentos incluem a falta de dinheiro para a compra; a falha na distribuição gratuita pelo serviço de saúde; as dificuldades de adaptação na tomada das medicações (dose, quantidade e horários) e a presença de efeitos colaterais indesejáveis. Tais questões podem ser evidenciadas nas frases temáticas a seguir:

O tratamento medicamentoso antihipertensivo foi descontinuo por falta de dinheiro para a compra de medicamentos $e$ devido a sua inexistência na Unidade Básica de Saúde;

A doente teve dificuldade ("uma calamidade") na adaptação de tomada de medicações (doses, quantidades, horários) necessitando de ajuda familiar para tal;

Durante dois anos a doente fez tratamento medicamentoso irregular na unidade de saúde de sua cidade (interior do Pará) devido aos efeitos colaterais dos medicamentos, além da dificuldade de seguir dieta hipossódica.

Com relação à dieta, as dificuldades para seguir o regime alimentar contendo restrições na ingestão de sal, gordura, carne e condimentos, foram associadas à "vontade de comer comida normal" e ao entendimento de que essas restrições dietéticas são como um "castigo", causando sentimento de "revolta". Tais dificuldades podem ser observadas através das seguintes frases temáticas:

O seguimento irregular da dieta hipossódica está associado à "vontade de comer comida normal, via todo mundo comendo normal, queria comer normal também";

A doente considera as restrições dietéticas (sal, gordura, carne, maionese, pimenta) como um "castigo", sente "revolta" por não poder ingerir "tudo o que mais você gosta" (comer, viajar, visitar os amigos).

As dificuldades quanto à acessibilidade ao serviço de saúde, ocorreram devido à inexistência de atendimento especializado, à demora paramarcar consulta ou retorno ao atendimento médico, à falta de recursos materiais, às falhas no atendimento médico com relação à detecção de complicações decorrentes da hipertensão arterial e à realização de tratamento sintomático nos momentos de crise hipertensiva. As dificuldades de acesso acabam por gerar distorções no serviço de saúde fazendo com que a porta de entrada para o atendimento se dê através do serviço de emergência ou pela relação pessoal de amizade entre médicos. As frases a seguir expressam estas questões:

O tratamento inicial foi em consultório privado e o acesso ao tratamento público (HC) foi por "amizade" entre os médicos;

$O$ atendimento de saúde no convênio médico da empresa não detectou problema renal decorrente da hipertensão arterial e realizou tratamento sintomático nos momentos de crise hipertensiva;

$O$ acesso ao tratamento no $\mathrm{HC}$ teve como porta de entrada o pronto-socorro, devido a piora da sintomatologia;

A não descoberta do problema renal "é perigosa" podendo levar à morte rápida;

A prescrição de medicamentos e o exame médico (medida da pressão arterial e a avaliação cardiaca) são considerados precários pelo doente por serem realizados por "jovens residentes";

$O$ atendimento de saúde no convênio médico realizou exames e tratamento sintomático nos momentos de crise hipertensiva $e$ não detectou outros problemas decorrentes da hipertensão arterial (complicação renal);

O acesso da doente ao tratamento num hospital público foi dificil (por não conseguir marcar consulta) e a porta de entrada acabou sendo o pronto-socorro.

O caminhamento ao $H C$ de São Paulo foi feito por falta de recursos (aparelho para arteriografia) no $H C$ de Goiânia e através de "amizade" entre os médicos.

MACIEL (1997) comprova semelhantes dificuldades no seguimento do tratamento da HA, citadas em nosso estudo: falta de dinheiro para comprar medicação, efeitos colaterais das drogas, o desconhecimento sobre a importância da tomada de medicação associando seu uso apenas com presença de sintomatologia. Os indivíduos foram inqueridos se havia sido prescrito o uso de anti- 
hipertensivo em consulta médica anterior e todos referiram afirmativamente. No entanto, somente $19,23 \%$ faziam o uso de medicamentos conforme a prescrição médica; 69,23\% faziam uso irregular e $11,54 \%$ abandonaram o tratamento. Com relação a estes dois últimos, a maior parte dos indivíduos eram do sexo feminino. A menor adesão das mulheres ao tratamento prescrito foi associada à sua familiaridade com sinais e sintomas das doenças por sempre ter tido o papel de cuidar de familiares, estando sujeitas a controlar a própria patologia. E, além disso, grande parte dos individuos relatou a presença de certos sinais e sintomas como parâmetro para a ingestão ou não de medicamentos. Outras dificuldades encontradas foram a ingestão de dieta hipossódica, como também a restrição alimentar; e, não gostar de marcar consulta médica indicando dificuldades na acessibilidade aos serviços de saúde; a má interpretação das orientações médicas e o conselho de leigos, indicando que os individuos hipertensos carecem de explicações detalhadas acerca do tratamento e dos medicamentos que deverão utilizar, bem como de noções básicas a respeito da hipertensão.

\subsubsection{Mudanças na vida dos doentes após o início do tratamento da hipertensão arterial}

As mudanças ocorridas na vida dos doentes, após o início do tratamento da hipertensão arterial, foram consideradas problemáticas e chegaram a provocar sentimentos/reações negativas:

As mudanças na vida, após o início do tratamento da hipertensão arterial, foram consideradas "problemas" para o doente devido: à presença de sintomatologia, ao inicio de tratamento medicamentoso, à perda do emprego e à ingestão irregular de dieta hipossódica;

As mudanças na vida da doente, após o início do tratamento da hipertensão arterial, provocaram "medo", "revolta", sentiu-se "desnorteada";

Houve muitas mudanças na vida da doente após o início do tratamento regular da hipertensão arterial, consideradas como tendo "acabado sua vida ... tudo que gosto".

O início do tratamento da hipertensão arterial, nem sempre coincidente com o período de conhecimento do diagnóstico, gerou mudanças na vida dos doentes, tais como: seguir regime alimentar, principalmente devido à necessidade de ingestão de dieta hipossódica; tomar grande quantidade de medicamentos; aparecimento de sintomatologia; deixar de trabalhar; interromper os estudos; deixar de viajar com a família e amigos; ficar longo período de tempo em internação hospitalar; ter que se aposentar; além de provocar alterações emocionais e físicas. As frases temáticas extraídas dos depoimentos dos doentes que indicam tais mudanças são:

Houve muitas mudanças na vida da doente após o início do tratamento da hipertensão arterial: fazer regime alimentar, tomar grande quantidade de medicamentos, deixar de viajar com a familia para lugares distantes sem recursos médicos, ter se aposentado.

As mudanças na vida da doente, após o início do tratamento da hipertensão arterial, foram: deixar sua casa, sua cidade e interromper os estudos, comer dieta hipossódica, ficar muito tempo internada, não viajar e sair de casa à noite com os amigos, deixar de trabalhar e alterações emocionais ("nervosa, irritada, raiva, discussões") e fisicas ("sono") decorrentes do uso da medicação.

Um dos doentes só modificou seu comportamento após o agravamento das complicações da hipertensão arterial:

O agravamento da sintomatologia, devido ao $2^{\circ}$ IAM e a complicação renal, além da pressão familiar, levou o doente a dar mais importância ao tratamento medicamentoso, dietético e à diminuição do tabagismo e do etilismo.

Um outro doente associa as modificações de comportamento ao seu amadurecimento pessoal, além do seu aprendizado durante a internação hospitalar:

O doente associa ao seu atual estado de amadurecimento ("psicológico") um dominio da hipertensão arterial através do medicamento e da sua mudança de comportamento ("ficar mais calmo");

Durante sua internação hospitalar, o doente refere ter aprendido a importância do controle de sal na alimentação.

MACIEL (1997) ao inquerir se a HA afetara a vida de hipertensos, 34,62\% responderam afirmativamente, $63,46 \%$ responderam negativamente e $1,92 \%$ não sabia se havia interferência. Os dados revelaram que a HA afetava mais as atividades sociais na vida das mulheres $(28,85 \%)$ em relação aos homens $(5,77 \%)$.

TRENTINI； SILVA； LEIMANN (1990) mencionam que pessoas com doenças crônicas enfrentam mudanças denominadas "novas incumbências", como: seguimento de uma ou mais 
modalidades de regime de tratamento (dieta alimentar, medicação, reabilitação fisica e repouso); conhecer sobre a doença; lidar com incômodos fisicos (aparecimento de sinais e sintomas, e referentes ao sono, eliminações, alimentação, integridade e energia física, respiração e alterações sensoriais). Outras mudanças ocorreram devido ao fato destes individuos terem enfrentado experiências de perdas nas relações sociais, financeiras, na capacidade física, nas atividades de lazer e perdas do prazer de fumar e beber. Além disso, os pacientes com doenças crônicas se depararam com ameaças à aparência individual, à vida e à preservação da esperança.

\section{SÍNTESE}

O grupo de hipertensos (6) participantes deste estudo caracterizou-se pela diversidade em relação à idade, ao sexo, à escolaridade, à renda per capta, à participação em entidades/grupos e às mudanças de ocupação e de renda devido à HA. Foi, no entanto, homogêneo quanto à sua dependência do serviço público de saúde para o seguimento do tratamento da HA, tanto no nivel de internação como no ambulatorial.

Essas diferentes características refletiramse nas reações/comportamentos dos hipertensos em relação ao seu cotidiano da vida após a descoberta ou o início do tratamento da HA. Alguns não modificaram a dinâmica de vida após a descoberta da HA; outros introduziram no dia a dia a tomada da medicação; e, outros relataram sofrimento com reações de revolta e raiva devidos às condições impostas pela doença às suas vidas.

A modificação ou não do cotidiano desses hipertensos, logo após a descoberta da HA, parece estar relacionada à presença de sintomatologia, à compreensão dos doentes sobre a doença e ao impacto desta nas suas vidas. Por outro lado, o início do tratamento da HA, nem sempre coincidente com a sua descoberta, também associado à presença de sintomatologia provavelmente decorrente de complicações da doença, foi relacionado a mudanças problemáticas na vida dos doentes, incluindo restrições alimentares, de lazer, de trabalho, de estudo e de convivio social.

Assim, o enfrentamento da cronicidade da HA envolve a compreensão do seu significado, pelos hipertensos, de acordo com suas concepções de saúde-doença, alterações emocionais e fisicas, além de outras dificuldades que interferem no seguimento do tratamento relacionadas à medicação, à dieta e à acessibilidade aos serviços de saúde.

Em sintese, conviver com a cronicidade da HA significa, ao longo do tempo, fazer/sofrer mudanças rio cotidiano da vida.

\section{REFERENCIAS BIBLIOGRÁFICAS}

BLACK, J.M.; MATASSARIN-JACOBS, E. Luckmann e Sorensen Enfermagem médico-cirúrgica: uma abordagem psicofisiológica. 4.ed. Rio de Janeiro, Guanabara Koogan, 1996. v.1. cap.9, p.117-33: Condições crônicas.

CAR, M.R. Da aparência à essência: a práxis assistencial dos trabalhadores da Liga de Hipertensão Arterial. São Paulo, 1993, 125p. Tese (Doutorado) - Escola de Enfermagem, Universidade de São Paulo.

CASTRO, V.D. de; CAR, M.R. Dificuldades e facilidades dos doentes no seguimento do tratamento da hipertensão arterial São Paulo, 1997, 34p. Monografia (Conclusão Curso de Graduação em Enfermagem) Escola de Enfermagem, Universidade de São Paulo.

CONSENSO BRASILEIRO DE HIPERTENSÃO ARTERIAL, III, Campos do Jordão, 1998.

FIORIN, J.L.; SAVIOLI, F.P. Para entender o texto: leitura e redação. São Paulo, Ática, 1991.

MACIEL, E.A.B. Estudo da adesão ao tratamento da hipertensão arterial. Ribeirão Preto, 1997, 212p. Dissertação (Mestrado) - Escola de Enfermagem de Ribeirão Preto, Universidade de São Paulo.

MENDES, I.A.C.; TREVIZAN, M.A. A necessidade de aprendizagem em pacientes crônicos. Enf. Atual, v.3, n. 18, p.4-'7, 1981.

PESSUTO, J. As necessidades básicas afetadas e os fatores de risco de clientes portadores de hipertensão arterial. Ribeirão Preto, 1994, 158p. Dissertação (Mestrado) - Escola de Enfermagem de Ribeirão Preto, Universidade de São Paulo.

SILVA, A.L.C.; COSTA, H.G.; RIZZO, L.M.G. O enfermeiro e o paciente em tratamento de longa duração. Rev. Bras. Enf., v.33, n.3, p.356-64, 1980 .

TRENTINI, M.; SILVA, D.G.V. da; LEIMANN, A.H. Mudanças no estilo de vida enfrentadas por pacientes em condições crônicas de saúde. Rev. Gaúcha Enf., v. 11, n.1, p.18-28, 1990.

WOOG, P. The chronic illness trajectory framework: the Corbin and Strauss nursing model. New York, Springer, 1992. 


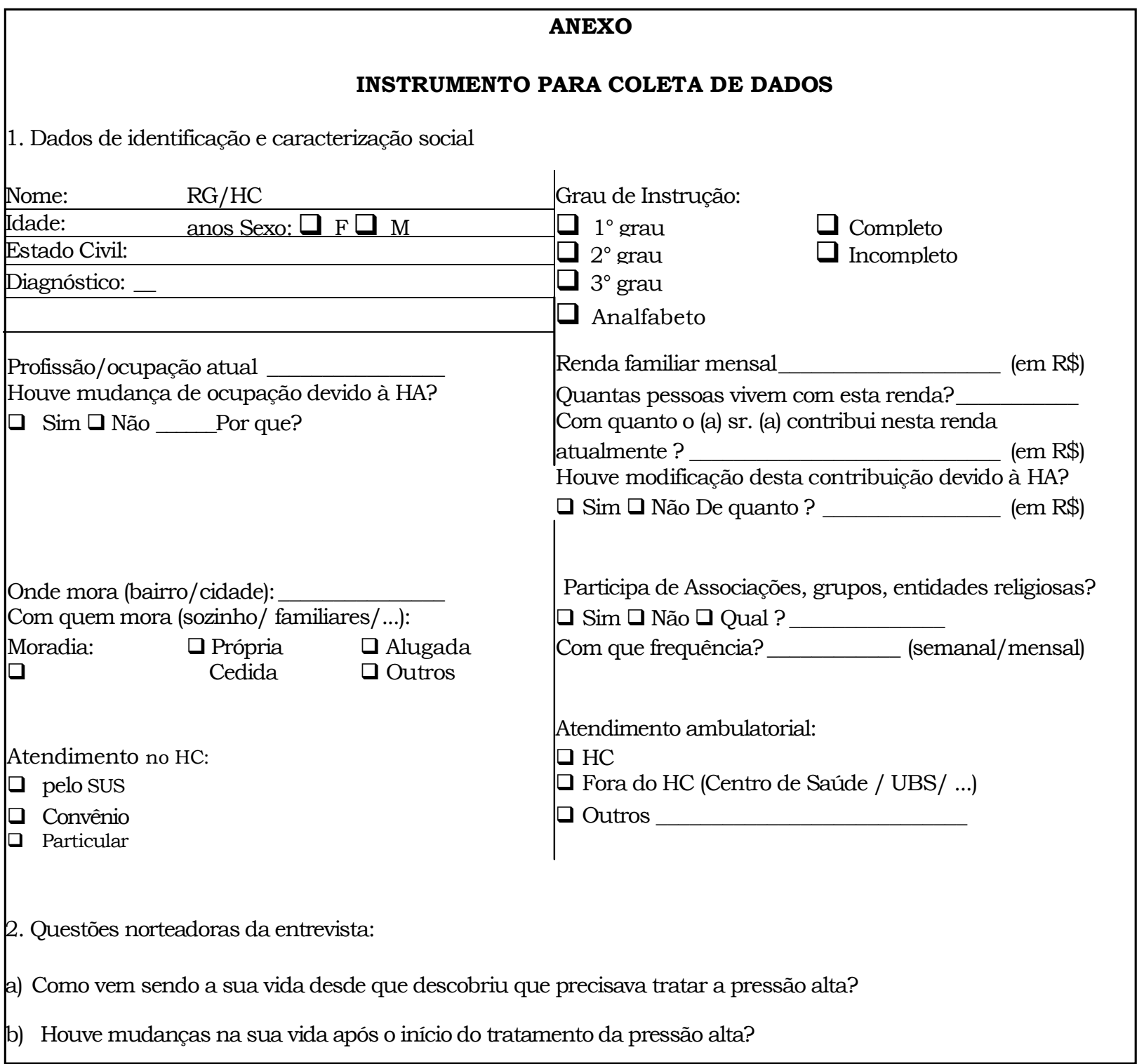

